\title{
Is EQ Boo a Quadruple System?
}

\author{
Igor M. Volkov ${ }^{1}$, Drahomír Chochol ${ }^{2}$, Natalia S. Volkova ${ }^{1}$ \\ and Igor V. Nikolenko ${ }^{3}$ \\ ${ }^{1}$ Sternberg Astronomical Institute, Moscow University, \\ Universitetsky Ave., 13, Moscow 119992, Russia \\ email: imv@sai.msu.ru \\ ${ }^{2}$ Astronomical Institute of the Slovak Academy of Sciences, \\ 05960 Tatranská Lomnica, Slovakia \\ email: chochol@ta3.sk \\ ${ }^{3}$ Scientific Research Institute, "Crimean Astrophysical Observatory," \\ Community Blue Bay, Shajn str., 1, Ukraine
}

\begin{abstract}
We present the precise multicolour photometry of the eclipsing variable EQ Boo $\left(\mathrm{P}=5^{d} .43, V=8^{m} .8\right)$, which is component "A" of the visual double star ADS 9422 $(\mathrm{F} 7 \mathrm{~V}+\mathrm{G} 0 \mathrm{~V}, \rho=1.3 ", \Delta \mathrm{mag}=0.7)$. From the analysis of these data, we can propose the existence of the fourth component with a late spectral type.
\end{abstract}

Keywords. eclipsing binary, multiple system, stellar evolution

\section{Introduction}

Otero et al. (2006) found the position of the secondary minimum of the eclipsing variable EQ Boo at the phase $\phi(I I)=0.399$, so its orbit is eccentric. We observed the star in 2007-2010 at the Zvenigorod, Crimean and Stará Lesná observatories. We obtained observations in two primary and three secondary minima. We always measured the brightness of both components of the visual double star together. Our techniques of the atmospheric extinction correction and the solution of the light curves were the same as those used in our previous papers: Volkov \& Volkova (2009), Volkov et al. (2010).

\section{Absolute parameters of the system}

After the solution of our $U B V R I$ light curves by the differential corrections method, we obtained absolute parameters of the system using Kepler's third law and the massluminosity relation for main sequence stars (see Table 1). If we accept that the third light in the system is only due to the component " $\mathrm{B}$ ", which is unresolved in our observations, then the parameters of the three stars do not satisfy the value of the common age $2.0 \cdot 10^{9}$ years. This contradiction can be resolved by assuming the existence of a fourth star with a later spectral type of $\mathrm{K} 2 \mathrm{~V}$ in the system (Fig. 1d). The details of our work are published in Volkov et al. (2011).

\section{Acknowledgements}

This research was partly supported by VEGA grants 2/0038/10 and 2/0094/11 (D. Chochol), and by Russian Foundation for Basic Research grant 11-02-01213-a (I. M. Volkov and N. S. Volkova). We used in our work the SIMBAD database of the Strasbourg center of astronomical data (France) and the ADS service of NASA (USA). 

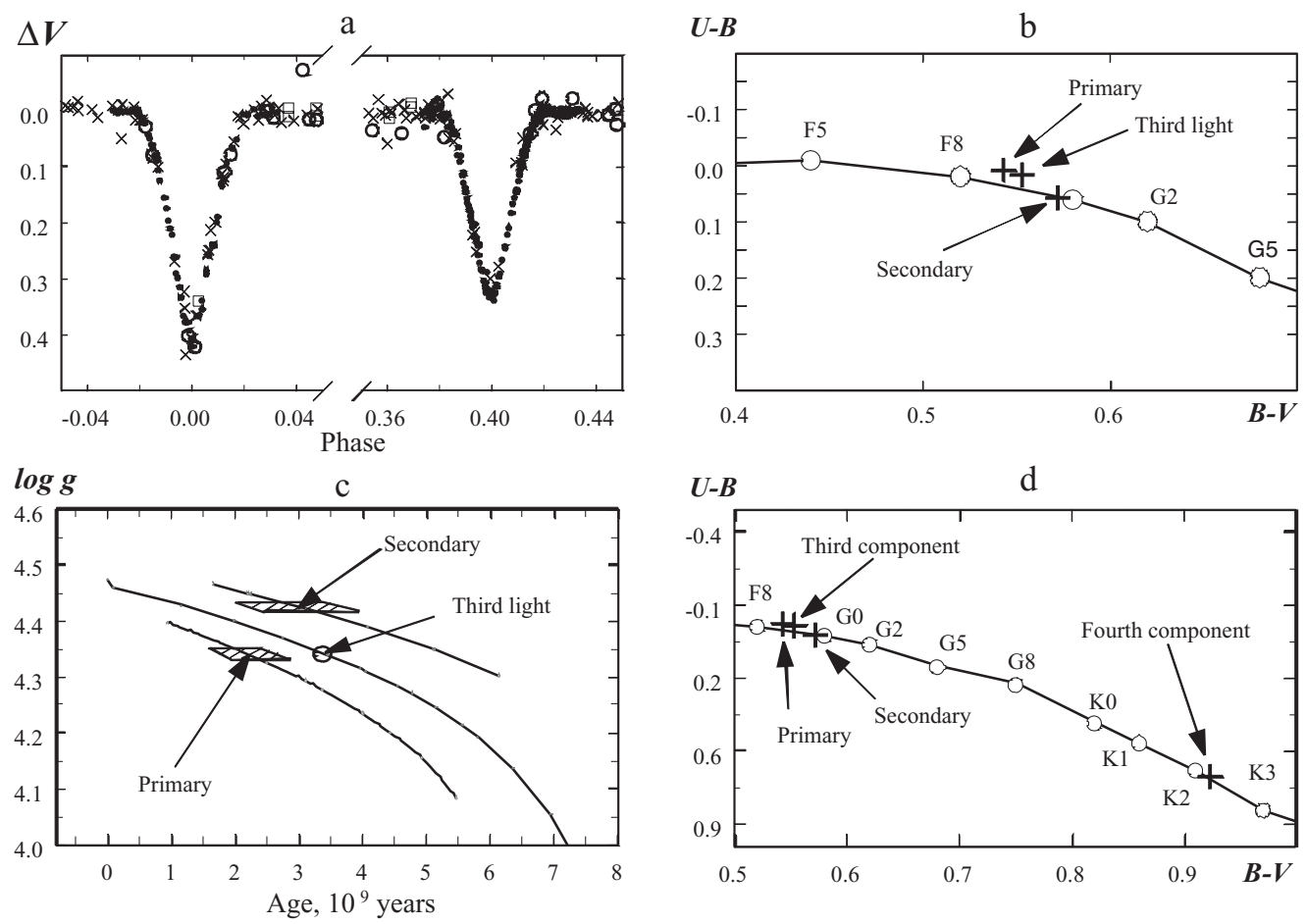

Figure 1. a) The light curve of EQ Boo (points - our $V$ photometry, crosses - ASAS (Pojmanski 2002), circles - Hipparcos (Perryman et al. 1997), squares - ROTSE (Woźniak et al. 2004)). b) The position of the components in the standard two-colour diagram. c) The evolutionary diagram for the stars with the masses from Table 1. d) The position of the components in the standard two-colour diagram taking into account the complicated structure of the third light.

Table 1. Absolute parameters of EQ Boo.

\begin{tabular}{|c|c|c|c|}
\hline Parameter & Primary & Secondary & The third light \\
\hline Mass, $\mathrm{M}_{\odot}$ & $1.15 \pm 0.05$ & $1.05 \pm 0.04$ & $1.11 \pm 0.07$ \\
Radius, $\mathrm{R}_{\odot}$ & $1.19 \pm 0.03$ & $1.03 \pm 0.03$ & $1.16 \pm 0.05$ \\
Luminosity, $\mathrm{L}_{\odot}$ & $1.45 \pm 0.08$ & $1.04 \pm 0.07$ & $1.26 \pm 0.08$ \\
$\mathrm{~T}_{\text {eff }}$ & $6120 \pm 100 \mathrm{~K}$ & $5980 \pm 80 \mathrm{~K}$ & $5940 \pm 80 \mathrm{~K}$ \\
Spectral type (from our $U B V$ data) & $\mathrm{F} 8 \mathrm{~V}$ & G0 V & G0 V \\
\hline
\end{tabular}

\section{References}

Otero, S. A., Wils, P., Hoogeveen, G., \& Dubovsky, P. A., 2006, IBVS, 5681, 1

Perryman, M. A. C., Lindegren, L., Kovalevsky, J., Hoeg, E., Bastian, U., Bernacca, P. L., Crézé, M., Donati, F., Grenon, M., van Leeuwen, F., van der Marel, H., Mignard, F., Murray, C. A., Le Poole, R. S., Schrijver, H., Turon, C., Arenou, F., Froeschlé, M., \& Petersen, C. S., 1997, $A \mathscr{E} A, 323, \mathrm{~L} 49$

Pojmanski, G., 2002, AcA, 52, 397

Woźniak, P. R., Vestrand, W. T., Akerlof, C. W., Balsano, R., Bloch, J., Casperson, D., Fletcher, S., Gisler, G., Kehoe, R., Kinemuchi, K., Lee, B. C., Marshall, S., McGowan, K. E., McKay, T. A., Rykoff, E. S., Smith, D. A., Szymanski, J., \& Wren, J., 2004, AJ, 127, 2436

Volkov, I. M. \& Volkova, N. S., 2009, Astron. Rep., 53, 136

Volkov, I.M., Volkova, N.S., \& Chochol, D., 2010 Astron. Rep., 54, 418

Volkov, I.M., Volkova, N.S., Chochol, D. \& Nikolenko, I.V., 2011 Astron. Rep., 55, 824 